\title{
Charcot Neuroarthropathy may be the RAGE
}

\author{
Kara A. Witzke ${ }^{1 *}$ and Aaron I. Vinik ${ }^{2}$
}

${ }^{1}$ Department of Kinesiology, California State University, San Marcos, CA, USA

${ }^{2}$ Strelitz Diabetes Centers and Neuroendocrine Unit, Eastern Virginia Medical School, Norfolk, US

\begin{abstract}
Charcot neuroarthropathy (CNA) is a degenerative bone disease of unknown origin that occurs in the ankle and midfoot. While not exclusive to patients with diabetes, CNA will develop in about $0.1-2.5 \%$ of those with diabetes $[1,2]$. Recent work has focused on understanding the common link between inflammation induced by oxidative stress and the ensuing accumulation of advanced glycation end products (AGEs) leading to impaired bone mineral matrix mineralization and reduced bone strength in CNA. Collagen cross-linking is important for bone strength but non-enzymatic crosslinking results in an accumulation of AGEs in bone, accelerated by oxidative stress. AGEs stimulate apoptosis of osteoblastic cells mediated through RAGE, the pattern recognition receptor for AGE. AGE-RAGE interactions alter intracellular signaling via pro-inflammatory cytokines that further propagate diabetic complications. SRAGE is a soluble form of RAGE that competes with membrane-bound RAGE receptors for AGEs and functions as a decoy to "mop up" circulating AGEs, preventing them from binding to RAGE and causing an inflammatory cascade. A reduction in serum SRAGE is associated with increased vascular risk factors in diabetes mellitus and the metabolic syndrome. Recently, it was shown that bone specimens removed from CNA patients display reduced trabecular number and the presence of woven bone. We have previously shown derangements in collagen structure of the Achilles tendon and reduced calcaneal bone stiffness in CNA patients correlated with a marked reduction in circulating SRAGE. These results suggest a relationship between impaired AGE defense, bone turnover, and reduced bone quality in CNA. Based on limited studies relating RAGE to bone status and a few animal studies using SRAGE administration to interfere with RAGE expression, we speculate that SRAGE administration may have potential to interrupt osteoclastic activation in those who lack adequate endogenous production such as patients with CNA.
\end{abstract}

\section{Introduction}

Charcot neuroarthropathy (CNA) is a progressive, degenerative process which usually occurs in the bones of the ankle and midfoot. While not exclusive to patients with diabetes, CNA will develop in about $0.1-2.5 \%$ of those with diabetes $[1,2]$ and has been reported in up to $13 \%$ of people with diabetes at a specialized diabetic foot and ankle clinic [3]. The precise incidence is not known, but it is estimated that over 375,000 patients in the United States alone are affected by CNA [4].

Acute clinical presentation is marked by localized redness, inflammation, possible pain, and joint and/or bone deformity. The underlying peripheral neuropathy that always accompanies CNA may make the condition go unnoticed due to a lack of pain sensation. Standard radiography in the early stage is often unable to distinguish CNA from other conditions and while radioisotope technetium (Tc$99 \mathrm{~m}$ ) bone scintigraphy has good sensitivity, it has poor specificity for showing osseous changes until later stages of the condition. Magnetic resonance imaging (MRI) has been shown to be a good technique for evaluating bony inflammation and localized soft tissue involvement in the early stage [5] but may over-estimate damage and can be difficult to assess in the presence of osteomyelitis. MRI has, however, been shown to correlate bone marrow edema with clinical evaluations such as soft tissue edema and/or pain [6]. During the acute phase, the CNA foot is hot with increased blood flow when measured by laser doppler techniques [7]. The chronic (quiescent) stage of CNA is characterized by absence of edema, erythema or warmth, and hypertrophic bone healing. Several systems exist for classifying CNA and are summarized in a concise clinical review by Slater et al. [8]. The following brief review will present what is currently known about the possible pathogenesis of CNA and will suggest some possible pathways that warrant further study.

\section{Mechanical Theories of the Pathogenesis of CNA}

While two theories were once thought to provide competing views of the cause of CNA, they are now accepted to both provide useful information about the pathogenesis of this condition. The "neurovascular theory" of CNA suggests that bony changes are the result of damage to the autonomic nervous system causing hyperemia and an increase in blood flow to the limbs due to arteriovenous shunts. The "neurotraumatic theory" postulates that in the presence of sensory neuropathy, CNA may be triggered by some external trauma to the foot that sets the inflammatory response into motion. In either case, the progression of localized inflammation eventually leads to bone lysis, microfracture and bone deformity. Interestingly these two pathways may be linked since it has become evident that autonomic dysfunction is associated with an increase in circulating inflammatory cytokines [9]. Recent studies support the notion that autonomic reflexes may control the inflammatory response [10].

Although each of these theories has merit, both fail to explain why CNA occurs in only a small percentage of those with neuropathy, why the condition is often unilateral, and how the inflammatory response

*Corresponding author: Kara A. Witzke, Ph.D, Department of Kinesiology, California State University, San Marcos, 333 S. Twin Oaks Valley Rd, San Marcos, CA 92009, USA, Tel: 760750 7355; Fax: 760750 3237; E-mail: kwitzke@csusm. edu

Received November 13, 2011; Accepted January 16, 2012; Published January 20, 2012

Citation: Witzke KA, Vinik AI (2012) Charcot Neuroarthropathy may be the RAGE. J Diabetes Metab S1:005. doi:10.4172/2155-6156.S1-005

Copyright: (c) 2012 Witzke KA, et al. This is an open-access article distributed under the terms of the Creative Commons Attribution License, which permits unrestricted use, distribution, and reproduction in any medium, provided the original author and source are credited. 
contributes to the progression of the disease. The work of Mabilleau and Edmonds [11] suggests that although osteoclastic resorption mediated by receptor activator for nuclear factor- $\kappa \mathrm{B}$ ligand (RANKL) occurs in acute CNA, a RANKL-independent pathway mediated by proinflammatory cytokines may also be important [11]. As a result, recent work has focused on understanding the common link between inflammation induced by oxidative stress and the ensuing accumulation of advanced glycation end products (AGEs) leading to impaired bone mineral matrix mineralization and reduced bone strength. As the authors have previously reported, the common link may indeed be the severity of autonomic neuropathy found in CNA [12]. This observation was cross sectional, however, and requires further elaboration.

\section{AGEs, collagen cross-linking, and bone strength}

Bone density and bone quality are both important, and arguably independent, factors in the determination of bone strength. Stabilization of newly formed bone collagen fibers is achieved by crosslinking between nearby collagen molecules. Collagen cross-linking is a determinant of bone quality due to its effects on tensile strength and post-yield properties of bone. Cross-links can form enzymatically by the action of lysyl oxidase or non-enzymatically, resulting in advanced glycation end products (AGEs). In contrast to the beneficial effects of enzymatic cross-linking processes on bone strength, AGEs cross-links have been shown to deteriorate both the biological and mechanical properties of bone [13]. Glycated collagen has an impaired ability to promote differentiation of preosteoblasts to mature osteoblasts in vitro [14] and impairs the adhesion of osteoblastic cells to the matrix [15]. Non-enzymatic cross-links AGEs in bone collagen make collagen fibers brittle, leading to an accumulation of fatigue damage over time, which results in increased bone fragility [16-18]. These cross-links are markedly increased in bone with both low and high levels of bone turnover via oxidation reactions and are accelerated by oxidative stress $[19,20]$.

\section{The AGEs-diabetes-bone connection}

Hyperglycemia increases protein glycation and causes a gradual accumulation of advanced glycation end products in body tissues. These AGEs form on intra- and extracellular proteins, lipids, and nucleic acids, in complex arrangements that lead to cross-linking. It has also been shown that an accumulation of AGEs in collagen tissue such as skin, measured by skin autofluorescence, correlates with severity of peripheral and autonomic nerve abnormalities in diabetes, even before being clinically manifest [21]. We, along with others, have also shown that CNA patients display significantly worse autonomic neuropathy (both sympathetic and parasympathetic) than diabetic or control subjects [12]. We suspect that this loss of sympathetic activity is responsible for the increase in skin blood flow we have observed in the affected foot of Charcot patients, possibly indicating an opening of shunt vessels that increase perfusion of the feet which may further increase osteoclastic activity and speed bone resorption [7].

Both immunohistochemical and biochemical studies have shown that carboxymethyllysine (CML)-modified proteins are AGEs that accumulate in tissues affected by diabetic complications, such as bone. AGEs have been shown to stimulate apoptosis of osteoblastic cells via CML collagen, one of the predominant AGEs found in bone and serum in individuals with low bone mass [22]. This process is mediated through RAGE, the pattern recognition receptor for AGE. Of the three known receptors for AGE, RAGE is of particular interest because it is found on many cell types, especially those affected by diabetes. RAGE is constitutively expressed but is induced by reactions known to initiate inflammation. Patients with diabetes often display elevated CML levels that can bind and activate RAGE signaling [23]. Furthermore, AGE-RAGE interaction creates a chronic cascade of inflammation and tissue injury. In a study comparing bone properties of RAGE ${ }^{-1}$ deficient vs wild type mice, Ding et al. [24] showed increases in bone mineral density and content, trabecular bone volume, bone quality and decreased osteoclast formation in RAGE knockout mice compared to controls, asserting the important role of RAGE in osteoclast formation and diabetes-induced bone destruction.

In homeostasis, RAGE is expressed at low levels but during biological stresses such as inflammation and diabetes, RAGE expression is greatly up regulated as a direct result of AGE accumulation. Many studies have now shown that AGE-RAGE interactions alter intracellular signaling via pro-inflammatory cytokines such as IL-6, TNF- $\alpha$, and free radicals that further propagate diabetic complications [24]. These cytokines may stimulate an increased activation of NF- $\kappa B$ ligand (RANKL) and lead to enhanced osteoclastogenesis and impaired matrix mineralization $[25,26]$ which predisposes bone to fracture. However, Mabilleau et al. [27] suggests that a RANKL-independent pathway also plays a role in the development of CNA. Specifically, they have shown that patients with CNA have 1.7 and 2.1 times higher numbers of CD14-positive monocyte cells than either diabetic patients without CNA or healthy controls. These increases were mirrored by a similar elevation in TNF- $\alpha$ in patients with CNA versus the other two groups. CD14-positive monocytes are the most potent of the monocyte subpopulations to transform into bone resorbing osteoclasts. They suggest that anti-TNF biological therapies may be useful therapeutic agents for controlling progression to CNA [28].

\section{AGEs and bone quality}

Historically, bone research involving diabetic patients has shown a paradoxical relationship whereby type 2 diabetes patients often display above average bone mineral density (BMD) but unusually high rates of fracture [29]. We now suspect, however, that type 2 diabetes may induce a condition of low bone quality, suggesting impaired material properties of bone. An increase in AGEs cross-links in diabetic bone may explain the discrepancy between density and strength although direct evidence does not yet exist to support this hypothesis.

In support of this idea, the authors have previously shown derangements in collagen structure of the Achilles tendon of Charcot patients, perhaps caused by accumulation of AGE cross-links [30,31]. A recent study by La Fontaine et al. [32] extends these findings to include an observation of reduced trabecular number and the presence of woven bone in bone specimens removed from the feet of patients with CNA undergoing corrective surgery. Woven bone is characterized by disorganized collagen fibers with a high cellular content and inconsistent mineral content. La Fontaine et al. [32] further described the CNA bone specimens as having osteoid-like trabeculae with surrounding inflammatory infiltrate dominated by lymphocytes and eosinophils. They further observed an increase in the number of Howship's lacunae indicating increased osteoclastic activity [32]. They conclude that the trabeculae in CNA patients appear to have poor quality characteristics compared with controls and diabetic patients without CNA. It is difficult, however, to distinguish 
characteristics attributable to the acute vs. chronic stage of the disease since they included a mixed sample of CNA patients. This is the first study, however, to qualitatively describe the histochemical properties of Charcot bone which further sets the stage for continued work in this important area.

In support of the observations of La Fontaine's group, the authors have studied bone quality in vivo in CNA patients using quantitative ultrasonography (QUS) of the calcaneus. This study showed a $36 \%$ reduction in calcaneal bone stiffness using QUS between stage 2 CNA patients and both healthy controls and diabetic patients without CNA, despite comparable bone mineral density in the feet and proximal femur using DXA [12]. We measured BMD of the feet in "hand mode" using DXA, which provides a better estimate of actual foot BMD rather than inferring this from hip, spine, or whole body BMD scans. Others have shown a reduced BMD of the acute Charcot foot using quantitative ultrasound [33] but QUS likely underestimates BMD in acute CNA since edema increases the time for transmission of sound waves and has previously been shown to decrease the estimation of BMD [34]. The only study to report on BMD in stage 3 CNA found decreased BMD with an average of 5 years following the initial CNA event [35]. Our previous findings and those of La Fontaine et al. [32] correlate CNA pathogenesis with bone quality, rather than bone density which may be a more important predictor of fracture than bone density alone [26]

\section{sRAGE: A Better Indicator of Charcot Susceptibility?}

sRAGE is a soluble form of RAGE that competes with membranebound RAGE receptors for AGEs and functions as a decoy to "mop up" circulating AGEs, preventing them from binding to RAGE and causing an inflammatory cascade (Figure 1). A reduction in serum sRAGE is associated with increased vascular risk factors in diabetes mellitus and the metabolic syndrome [36]. It is also decreased in chronic inflammatory diseases including atherosclerosis, renal failure and aging [37], and in patients with Grade 5 (Wagner classification) diabetic neuropathy in the feet [38]. El-Mesallamy et al. [38] showed that serum sRAGE was elevated in diabetic patients without severe complications in the feet but reduced in those with the most severe diabetic foot condition (characterized by ulceration, infection, and gangrene infection). We have also recently reported a significant reduction of sRAGE in patients with chronic (quiescent) Charcot neuroarthropathy. In our study, sRAGE levels in CNA were reduced by $86 \%$ compared to healthy controls. We also showed that type 2 diabetic patients without CNA displayed a 50\% reduction in sRAGE compared to controls [12], which is in contrast to the results of El-Mesallamy [38] who reported elevated sRAGE in diabetic patients without diabetic foot compared to controls. These discrepancies may well be explained

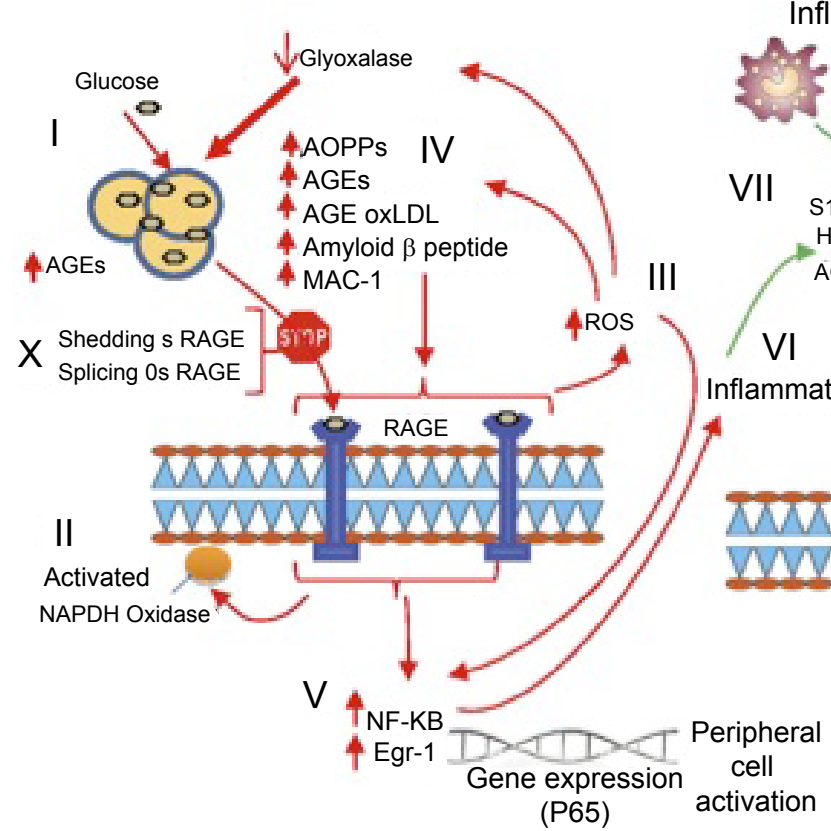

Inflammatory

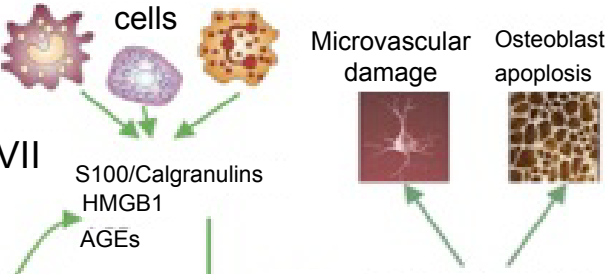

IX

Magnified Cellular Stress and Tissue Damage

Figure 1: The relationship between binding of ligands to the pattern recognition AGE receptor, RAGE and inflammation, gene expression, oxidative and nitrosative stress, and damage to the macro- and microvasculature. Elevated levels of glucose bind to proteins and form AGEs (I), which bind to RAGEs. RAGE signaling activates NADPH oxidase (II) and production of reactive oxygen species (ROS) (III). Increased ROS increases advance oxidation protein products (AOPPs), more AGEs, and AGE-modification of oxidized LDLs (oxLDLs). Furthermore, increased ROS may deplete glutathione, thereby suppressing glyoxalase I activity, a mechanism favoring further AGE accumulation (IV). AGEs, AOPPs, macrophage glycoprotein (MAC-1), and AGE-oxLDL ligands of RAGE sustain stimulation of RAGE, and these processes, together with increased ROS, activate key transcription factors such as nuclear factor-kB (NF-kB) and Egr-1 (V), which increase gene transcription factors and activate inflammatory mechanisms (VI). Consequences include increased migration and activation of RAGE-expressing neutrophils, monocytes/macrophages, T-cells, and dendritic cells (VII). This results in the release of the proinflammatory RAGE ligands S100/calgranulins and high-mobility group protein box-1 (HMGB1). In this inflammatory environment, further AGEs may be formed as well. Via interaction with RAGE, these ligands magnify activation of NF-KB, Agr-1, and other factors (VIII), thereby amplifying cellular stress and tissue damage leading to neurovascular and bone dysfunction. Soluble RAGE (sRAGE) formed from cleavage of RAGE by disintegrins such as ADAM 10, a metalloproteinase, and $\beta$ - and $y$-secretases. SRAGE or a spliced variant (esRAGE) compete for binding of ligands to RAGE, and a deficiency could theoretically initiate the sequence of events activating an inflammatory cascade with an increase in the expression of proinflammatory cytokines (E-selectin, endothelin-1 tissue factor, vascular endothelial growth factor, and other proinflammatorycytokines [interleukin-6 and tumor necrosis factor- $\alpha$ ]) and damage to neurons, kidney, eye, the vasculature, and even bone. Increasing sRAGE or its administration could competitively reduce activation of the AGE/RAGE pathway and it consequences, reproduced with permission from [42]. 
by the specificity of the antibodies used in the assays of sRAGE and the cross reaction of some with esRAGE leading to falsely elevated levels of sRAGE. We are the first, however, to correlate a reduction in sRAGE to bone outcomes in CNA. We have shown significantly lower bone stiffness but significantly higher osteocalcin levels in CNA. Furthermore, there was a positive correlation between calcaneal bone stiffness index and sRAGE $(\mathrm{r}=0.35, \mathrm{p}<0.01)$ and a negative correlation between bone stiffness index and osteocalcin $(r=-0.39, p<0.001)$. These results suggest a relationship between impaired AGE defense, increased bone turnover, and reduced bone quality in Charcot neuroarthropathy. We further hypothesize that CNA patients may be susceptible to AGEmediated osteoblast apoptosis which limits their ability to repair bone.

Animal studies also support the idea that RAGE plays a critical role in bone loss via stimulation of an inflammatory cascade. Lalla et al. [39] blocked RAGE using sRAGE as a decoy receptor for AGEs and showed diminished diabetic alveolar bone loss in periodontal disease in mice. Likewise, diabetic mice administered sRAGE display suppressed development of atherosclerotic lesions without a concomitant decrease in $\mathrm{HbAlc}$, cholesterol, or triglyceride. The authors speculate that diminished AGE formation and accumulation was due to the suppression of RAGE-mediated oxidative stress [40]. It will be of considerable interest to extend these animal studies to man creating a novel prospect for treatment of this devastating disease.

Based on the small accumulation of animal studies and a few human studies relating RAGE to bone status, and the few animal studies using sRAGE administration to interfere with RAGE expression, it seems reasonable to assume that sRAGE administration has potential to interrupt osteoclastic activation in those who lack adequate endogenous production such as patients with Charcot neuroarthropathy. We anticipate that future studies will clearly identify whether therapeutic administration of sRAGE or other low molecular weight RAGE antagonists have the ability to dramatically alter the course of diabetic complications induced by oxidative stress.

\section{References}

1. Sinha S, Munichoodappa CS, Kozak GP (1972) Neuro-arthropathy (Charco joints) in diabetes mellitus (clinical study of 101 cases). Medicine (Baltimore) 51: $191-210$

2. Myerson M (1996) Salvage of diabetic neuropathy arthropathy with arthrodesis, in Surgery of disorders of the foot and ankle, Helal B, Cracchiolo A, Myerson M, Editor 1996, Martin Dunitz: London. 513-522.

3. Armstrong DG, Todd WF, Lavery LA, Harkless LB, Bushman TR (1997) The natural history of acute Charcot's arthropathy in a diabetic foot specialty clinic. J Am Podiatr Med Assoc 87: 272-278.

4. Childs M, Armstrong DG, Edelson GW (1998) Is Charcot arthropathy a late sequela of osteoporosis in patients with diabetes mellitus? J Foot Ankle Surg 37: $437-439$

5. Chantelau E, Poll LW (2006) Evaluation of the diabetic charcot foot by MR imaging or plain radiography--an observational study. Exp Clin Endocrinol Diabetes 114: 428-431.

6. Schlossbauer T, Mioc T, Sommerey S, Kessler SB, Reiser MF, et al. (2008) Magnetic resonance imaging in early stage charcot arthropathy: correlation of imaging findings and clinical symptoms. Eur J Med Res 13: 409-414.

7. Shapiro SA, Stansberry KB, Hill MA, Meyer MD, McNitt PM, et al. (1998) Normal blood flow response and vasomotion in the diabetic Charcot foot. $\mathrm{J}$ Diabetes Complications 12: 147-153.

8. Slater RA, Ramot Y, Buchs A, Rapoport MJ (2004) The diabetic Charcot foot. Isr Med Assoc J 6: 280-283.
9. Lieb DC, Parson HK, Mamikunian G, Vinik Al (2012) Cardiac autonomic imbalance in newly diagnosed and established diabetes is associated with markers of adipose tissue inflammation. Exp Diabetes Res 2012: 878760.

10. Tracey I (2011) Can neuroimaging studies identify pain endophenotypes in humans? Nat Rev Neurol 7: 173-181.

11. Mabilleau G, Edmonds ME (2010) Role of neuropathy on fracture healing in Charcot neuro-osteoarthropathy. J Musculoskelet Neuronal Interact 10: 84-91.

12. Witzke KA, Vinik AI, Grant LM, Grant WP, Parson HK, et al. (2011) Loss of RAGE defense: a cause of Charcot neuroarthropathy? Diabetes Care 34 1617-1621.

13. Vashishth D (2007) The role of the collagen matrix in skeletal fragility. Curr Osteoporos Rep 5: 62-66.

14. Katayama Y, Celic S, Nagata N, Martin TJ, Findlay DM (1997) Nonenzymatic glycation of type I collagen modifies interaction with UMR 201-10B preosteoblastic cells. Bone 21: 237-242.

15. McCarthy AD, Uemura T, Etcheverry SB, Cortizo AM (2004) Advanced glycation endproducts interefere with integrin-mediated osteoblastic attachment to a type-I collagen matrix. Int J Biochem Cell Biol 36: 840-848.

16. Vashishth D, Gibson GJ, Khoury JI, Schaffler MB, Kimura J, et al. (2001) Influence of nonenzymatic glycation on biomechanical properties of cortical bone. Bone 28: 195-201.

17. Saito M, Mori S, Mashiba T, Komatsubara S, Marumo K (2008) Collagen maturity, glycation induced-pentosidine, and mineralization are increased following 3-year treatment with incadronate in dogs. Osteoporos Int 19: 13431354.

18. Hernandez CJ, Tang SY, Baumbach BM, Hwu PB, Sakkee AN, et al. (2005) Trabecular microfracture and the influence of pyridinium and non-enzymatic glycation-mediated collagen cross-links. Bone 37: 825-832

19. Saito M, Fujii K, Marumo K (2006) Degree of mineralization-related collagen crosslinking in the femoral neck cancellous bone in cases of hip fracture and controls. Calcif Tissue Int 79: 160-168.

20. McCarthy AD, Etcheverry SB, Bruzzone L, Lettieri G, Barrio DA, et al. (2001) Non-enzymatic glycosylation of a type I collagen matrix: effects on osteoblastic development and oxidative stress. BMC Cell Biol 2: 16.

21. Meerwaldt R, Links TP, Graaff R, Hoogenberg K, Lefrandt JD, et al. (2005) Increased accumulation of skin advanced glycation end-products precedes and correlates with clinical manifestation of diabetic neuropathy. Diabetologia 48 1637-1644.

22. Hein G, Weiss C, Lehmann G, Niwa T, Stein G, et al. (2006) Advanced glycation end product modification of bone proteins and bone remodelling: hypothesis and preliminary immunohistochemical findings. Ann Rheum Dis 65: 101-104

23. Schmidt AM, Yan SD, Yan SF, Stern DM (2001) The multiligand receptor RAGE as a progression factor amplifying immune and inflammatory responses. $\mathrm{J}$ Clin Invest 108: 949-955.

24. Ding KH, Wang ZZ, Hamrick MW, Deng ZB, Zhou L, et al. (2006) Disordered osteoclast formation in RAGE-deficient mouse establishes an essential role for RAGE in diabetes related bone loss. Biochem Biophys Res Commun 340: 1091-1097.

25. Macaione V, Aguennouz M, Rodolico C, Mazzeo A, Patti A, et al. (2007) RAGE-NF-kappaB pathway activation in response to oxidative stress in facioscapulohumeral muscular dystrophy. Acta Neurol Scand 115: 115-121.

26. Yaturu S (2009) Diabetes and skeletal health. J Diabetes 1: 246-254.

27. Mabilleau G, Petrova NL, Edmonds ME, Sabokbar A (2008) Increased osteoclastic activity in acute Charcot's osteoarthropathy: the role of receptor activator of nuclear factor-kappaB ligand. Diabetologia 51: 1035-1040.

28. Mabilleau G, Petrova N, Edmonds ME, Sabokbar A (2011) Number of circulating CD14-positive cells and the serum levels of TNF-alpha are raised in acute charcot foot. Diabetes Care 34: e33.

29. Vestergaard P (2007) Discrepancies in bone mineral density and fracture risk 
in patients with type 1 and type 2 diabetes--a meta-analysis. Osteoporos Int 18: $427-444$

30. Vashishth D (2009) Advanced glycation end-products and bone fractures. IBMS BoneKey 6: 268-278.

31. Grant WP, Sullivan R, Sonenshine DE, Adam M, Slusser JH, et al. (1997) Electron microscopic investigation of the effects of diabetes mellitus on the Achilles tendon. J Foot Ankle Surg 36: 272-278.

32. La Fontaine J, Shibuya N, Sampson HW, Valderrama P (2011) Trabecular quality and cellular characteristics of normal, diabetic, and charcot bone. J Foot Ankle Surg 50: 648-653.

33. Petrova NL, Edmonds ME (2010) A prospective study of calcaneal bone minera density in acute Charcot osteoarthropathy. Diabetes care 33: 2254-2256.

34. Johansen A, Stone MD (1997) The effect of ankle oedema on bone ultrasound assessment at the heel. Osteoporosis international : a journal established as result of cooperation between the European Foundation for Osteoporosis and the National Osteoporosis Foundation of the USA 7: 44-47.

35. Greenhagen R, et al. Peripheral and central bone mineral density in Charcot neuroarthropathy compared to diabetic and nondiabetic population. J Am Podiatr Med Assoc. (in press).

36. Humpert PM, Djuric Z, Kopf S, Rudofsky G, Morcos M, et al. (2007) Soluble
RAGE but not endogenous secretory RAGE is associated with albuminuria in patients with type 2 diabetes. Cardiovasc Diabetol 6: 9

37. Maillard-Lefebvre $\mathrm{H}$, Boulanger E, Daroux M, Gaxatte C, Hudson BI, et al. (2009) Soluble receptor for advanced glycation end products: a new biomarker in diagnosis and prognosis of chronic inflammatory diseases. Rheumatology (Oxford) 48: 1190-1196.

38. El-Mesallamy HO, Hamdy NM, Ezzat OA, Reda AM (2011) Levels of Soluble Advanced Glycation End Product-Receptors and Other Soluble Serum Markers as Indicators of Diabetic Neuropathy in the Foot. J Investig Med 59: 1233 -1238.

39. Lalla E, Lamster IB, Feit M, Huang L, Spessot A, et al. (2000) Blockade of RAGE suppresses periodontitis-associated bone loss in diabetic mice. J Clin Invest 105: 1117-1124.

40. Park L, Raman KG, Lee KJ, Lu Y, Ferran LJ, et al. (1998) Suppression of accelerated diabetic atherosclerosis by the soluble receptor for advanced glycation endproducts. Nat Med 4: 1025-1031.

41. Yan SF, Ramasamy R, Schmidt AM (2010) The RAGE axis: a fundamenta mechanism signaling danger to the vulnerable vasculature. Circ Res 106: 842 853.

42. Vinik A (2011) The question is, my dear watson, why did the dog not bark?: the joslin 50-year medalist study. Diabetes Care 34: 1060-1063.
This article was originally published in a special issue, Diabetic Osteoporosis handled by Editor(s). Dr. Laura McCabe, Michigan State University, USA 